\title{
The Methodological Implications of Resolving the Realism Debate
}

\author{
Dan McArthur
}

This paper explores the question of whether or not the resolution of the realism question has any implications for scientific methodology. It proposes a solution to the question that draws features from recent "deflationary" approaches to the realism question. The normative methodological role of the deflationary approach is then defended from the claim that no interpretative, normative or methodological role is left for a deflationary position. An illustration of the utility of the approach is demonstrated through a case study of the role that the realism question has played in the field of quantum mechanics.

Keywords: realism, deflationary metaphysics, Quantum theory

In recent years the debate over scientific realism has taken a "deflationary" turn. Many philosophers of science, largely following the lead of Arthur Fine, have abandoned the quest for a global solution to the question that is supposed to apply to the whole of science. This paper examines some of the implications of this deflationary turn and, more specifically, examines the question of the normative role that it leaves for the philosophy of science. This examination is undertaken in two main parts.

The first section draws from the writings of Arthur Fine and some recent work by Sergio Sismondo and others in order to develop a plausible deflation- ary position. The nature of a plausible deflationary position is important to clarify because not all the current deflationary positions are identical. One question that needs to be clarified is whether or not a deflationary position simply identifies a general non-philosophical position common to all of the sciences (as Fine argues) or if it avoids a general $a$ priori stance to the realism question but finds different stances to it in the different sciences. Another important question that is addressed is the degree to which any deflationary position can be normative with regards to a science's interpretative stance.

Fine's (1986: 177) own deflationary 
position, The Natural Ontological Attitude (NOA), in his view, adds nothing to science but simply takes it on its own terms. If this is so, then any normative or methodological role that the philosophy of science might have for the practice of science is eliminated. This is because Fine bans any "philosophical additives" to science and demands that it be taken at face value. Taken this way, science, Fine argues, can be seen to espouse no philosophical theory at all either realism or anti-realism. Sismondo's deflationary approach, on the other hand, avoids NOA's general ban on philosophical interpretations and leaves it to the philosopher to account for science in terms of the stances that are found in specific scientific programs. I accept this conclusion, but I argue (drawing on some recent "naturalist" philosophies of science) that there is also a normative role for philosophy in the adjudication of the correct stance on the realism debate, on a case by case basis. The first section thus shows that a deflationary approach to the realism question can indeed have normative import in so far as a science's stance on the realism question has an effect on its general methodological values (i.e. what it takes as its general goal: causal explanation or empirical adequacy).

The second part of this paper illustrates the conclusions developed earlier through a case study of the role of the realism question in the practice of quantum mechanics. I propose, following the results of this case study, that a properly conceived deflationary approach to the realism question does have a substantial normative role to play in identifying the better alternative with regards to general methodological values. Before proceeding to develop the arguments mentioned above, however, it is worthwhile to consider the general background to the realism question and define the ways in which it is important to the themes of this paper.

Scientific realism has been the received view in the philosophy of science since the 1960's, it is the view that the constituents of scientific theories, including theoretical posits that cannot be observed, correspond to real structures in nature. The opposing position, antirealism, contends that unobservables are not taken to exist but should be viewed simply empirically consistent with what is observed in experiments. Anti-realism was revived by the work of van-Frassen (1980) who defends a form of anti realism which contends that realism is untenable because any number of different theoretical models can be consistent with a set of observations and that no way exists to tell which one is the "real" model. As I have noted above, recent literature has taken a "deflationary" turn toward positions that seek a compromise between realism and anti-realism.

The debate has considerable implications for the philosophy of science's traditional normative role, i.e. its task to describe not only the nature of the knowledge that science provides, but the sort of knowledge that it ought to provide and the way it ought to proceed in providing it. Realists have traditionally seen the goal of science as the true description of nature and characterised scientific knowledge as corresponding to nature. This entails, for any given science, complete causal descriptions that explain the phenomena being studied. Anti-realists, like van Fraassen, see the 
goal of science (and view scientific knowledge) as the construction of models that are consistent with experimental findings for the purposes of generating predictions. Anti-realists see the complete causal descriptions demanded by the realist as unnecessary so long as a given model produces successful predictions. In the case of equally accurate models, for the anti-realist, the choice between them ought to be made on the basis of criteria that do not relate to truth: simplicity, mathematical elegance and so on.

These normative prescriptions on what the scientific knowledge and goals of science ought to be like have an effect on a science's general methodological values. What are taken to be the general goals of science will effect whether or not a theory is regarded as complete. They will also effect the way a theory is constructed and deployed, in so far as they dictate what a theory ought to be about. A realist will regard a theory that makes no attempt at causal explanation of structures that underlie experimental observations as incomplete regardless of the accuracy of its predictions, whereas an anti-realist takes the opposite view.

The normative role of the realism question has, for example, played a large role in foundational debates that have attended quantum mechanics for most of this century. For this reason the realism debate in quantum theory will serve as the basis for the case study that will comprise the second part of this paper. It is, however, worthwhile to set out some of the central issues in order to clarify exactly what is at stake before proceeding to more detailed discussion of recent turns in the general realism debate.
One striking feature of quantum mechanics is the great accuracy of its predictions. In fact, the mainstream interpretation of quantum mechanics takes the theory to be a mathematical device used for calculating predictions, making no reference to causal processes that take place between the preparation of a system and the final measured results. The general methodological approach in mainstream quantum mechanics, then, has been to seek mathematical models that refine predictive accuracy and to avoid digressions that seek to explain why the models work. Realists have tended to insist that this approach leaves quantum mechanics incomplete and in need of replacement by theories that meet the requirement for complete descriptive explanation. These alternatives are generally referred to as "hidden variable" theories. Much fundamental quantum research has revolved around the testing of hidden variable theories, and to date they have met with little success. The most famous of such experiments to test hidden variable accounts is the so-called EPR (Einstein, Podolsky, Rosen) paradox. This work will be discussed in detail at a later point. However, it is important to point out that the debating camps in the EPR debate take the positions that they do on the basis of quite different sets of general methodological values on the basis of their position on the realism debate. Realists have insisted that quantum mechanics should proceed by searching for causal explanations (that include unobservable structures) while anti-realists regard the construction of such explanations as unnecessary, claiming that the production of accurate predictive models is all that is required. 
Recently, many philosophers of science have argued that science is too diverse to be accurately described as being either completely realist or anti-realist. They argue for various "deflationary" positions that avoid the global application of either realism or anti-realism. The general approach recommended by such positions is to read science's position on the realism question from science itself instead of imposing an $a$ priori philosophical position. This raises questions concerning philosophy's traditional normative role, if science's philosophical position is simply read from science itself then philosophy's role must be seen as merely descriptive. If this is so, how can philosophy provide any normative account of what science ought to do, given that philosophy's role is simply to describe what science is like? It is this question that will be subsequently addressed.

\section{NOA and the "Deflationary" Approaches}

The paradigm case of a deflationary philosophy of science is Arthur Fine's attempt to sketch a compromise position between realism and anti-realism, a position he dubs "the Natural Ontological Attitude" or NOA. The Basic tenets of NOA are straightforward. NOA eschews both the realist and anti-realist push to attribute philosophical interpretations to scientific truth claims and claims to take at face value what it claims is actually found in science. For the realist a belief in a claim like "there are electrons" implies that this statement corresponds to the "real world". For the anti-realist this statement means that the statement coheres with empirical data and no cor- respondence is implied. For NOA both claims are problematic because they both impose a philosophical theory of truth on the scientific statement. NOA adopts a much more modest approach to truth, adopting Tarski's referential semantics which (in Fine's reading) simply state that claiming the sentence "the sentence 'snow is white' is true" is warranted in those situations when one is warranted in saying "snow is white". The term "truth" adds nothing to the statements that are true. Thus for NOA the statement "there are electrons" just means what it says, "there are electrons" and there is "nothing more to say" (Fine, 1986: 134).

Despite its claim to take science at face value and avoid philosophical additions to the interpretation of scientific practice, NOA fails in its goal of analysing science within its own context. Sismondo (1997:222) argues that the attention to scientific practice urged by NOA can lead us to "metaphysical" (i.e. philosophically interpretative) questions, "some attention to practice can take us away from metaphysics, but only a little more can lead us right back". According to Sismondo when certain specific instances of scientific practice are analysed closely it becomes clear that they are best understood in terms of specific interpretations and it is indeed possible to arrive at the best such interpretation on a case by case basis. Crasnow (2000: 126) defends similar conclusions and recommends that we replace NOA with a "philosophical attitude" that recognises the plurality within science. However, unlike Sismondo she does not develop the methodological implications of her view.

NOA tries to formulate a compromise 
position between realism and anti-realism, attributing to each a shared core position that takes scientific truths at face value. What NOA is specifically deflationary about, then, is just the theory of truth that should be adopted, favouring the supposedly 'neutral' disquotational theory. However, in terms of its conception of scientific practice, according to Sismondo (1997: 222), NOA conceives of scientific practice in a realist style, "reading the book of nature". He contends that this is already going to far for it fails to appreciate the fact that a great deal of science "does not participate in a straightforwardly realist style" (Sismondo, 1997: 221).

Sismondo contends that focusing on the constructive nature of experimental practice can illustrate this point clearly. However, he is not arguing for the general adoption of a constructivist theory of truth that takes the world to be shaped by “consensus". Rather, he considers experiments in this way "only to emphasise scientists' contributions to knowledge through their [experimental] work" (Sismondo, 1997: 222). In this view, experimental knowledge is about a world that is, at least in part, constructed by scientific practice. This is so for reason that objects of experimentation are examined in highly artificial 'ideal' conditions in 'pure states' that are constructed in the laboratory and are greatly alienated from anything observed in nature.

Sismondo illustrates this claim by engaging in a detailed study of Prof. John Paul Scott's research program that studied the social behaviour in laboratory mice. Scott minimised the behavioural effects of genetic anomaly in his mice populations by using mice that had been bread to be more or less genetically iden- tical. In order to isolate the social variables that he was interested in, Scott placed his mice in artificial laboratory environments that were greatly abstracted from the natural environments within which wild mice are usually found. Sometimes there were no females, sometimes it was impossible for the mice to find hiding places and so on. For Sismondo (1997: 222), this programme is best understood in terms of constructivist metaphors. From the point of view of this constructivist philosophy of science, reality "co-responds" with science, science and nature shape each other in experimental practice. In the case of Scott's programme the reality studied by Scott was a constructed reality wherein the objects of his research were also products of its practice.

Sismondo, as we have seen, is clear that he is not arguing for the general adoption of constructivism. He instead argues that NOA's ban on interpretative "additions" to characterise scientific practice fails to do justice to certain instances of scientific practice which are best understood in terms of the metaphors provided by one or another interpretative stance. To paraphrase a wellknown metaphor, NOA's difficulties and the difficulties shared by realism and anti-realism are two sides of the same dubious coin. While realism and antirealism fail because they seek to impose their own interpretation universally on the whole of science, NOA fails because it seeks to impose its ban on interpretation universally. For Sismondo then, deflationary philosophies of science ought to be pluralistic with regard to interpretative stances. Some sciences are best understood in realist terms, some are best understood in constructivist terms, 
some positivistic etc. A position that follows Sismondo's then takes a pluralistic stance with regards to the interpretation of particular scientific research programmes. It also eschews a unified picture of science with regards to the realism question. Moreover, as the Scott case demonstrates (with the methodological role of its constructive stance), the deflationary approach recognises the methodological role the interpretations play. I will develop this deflationary position further by setting it in the context of the literature that deals with "naturalised philosophy of science".

\section{Deflationary Metaphysics and Normative Naturalism}

NOA, Sismondo's deflationary metaphysics and the variation of it that I am sketching can be compared in many respects to the ideas propounded in the literature dealing with "naturalised" philosophy of science. Naturalised philosophy of science (characterised here by the work of David Stump and Larry Laudan) draws essentially on the idea that the goals and methods of the philosophy of science ought to be informed by the goals and methods of science itself. It must be noted at the outset of such a comparison that this literature takes as its main focus the question of how philosophy should use the methods of science as its model to analyse science, it does not focus specifically on the realism question. Nevertheless this work is relevant to the discussion of this paper because if philosophy's approach to science has normative implications for science then this includes the proper stance to the realism question.

The stance to the realism question taken by a scientist (or by a philosopher making recommendations) does have methodological import, consider scientific realism's demand for causal explanation or, in contrast, van Fraassen's anti-realist recommendations about the methodological priority of "empirical adequacy". As David Stump has noted, however, naturalised philosophy of science has given rise to questions about the possibility of a normative role for the philosophy of science. Noting what science does do in no way implies what science ought to do. In essence this is a point which NOA might be said to fully adopt with its "hands off" approach to science on the part of the philosophy of science. Thus a very general question faces a deflationary philosophy or a naturalist one: how can normative judgements be applied to the methods, goals or interpretations of science if it is taken naturalistically without, at the same time, committing the naturalistic fallacy?

Furthermore, adopting a philosophy of science that tries to take science as its operating model to identify goals and methods can lead one into problems. Which science best serves as the best source for the philosophy of science? Taking any one particular model of scientific operation can defeat the purpose of naturalism by failing to account for the goals and methods of many sciences other than the one that serves as the general model. This is so because relying on the approach of one particular science to characterise scientific practice generally amounts to the same thing as trying to impose an $a$ priori philosophical interpretation. Both see science as a unified endeavour and seek to extend their interpretation across the whole domain 
of science and both ignore the very real plurality that exists within science. This can lead to the production of a picture of science that is just as distorted as the traditional a priori models of science that naturalism seeks to replace. This is indeed one way of looking at the failure of both NOA and also, for example, of van Fraassen's anti-realism; each while claiming to learn from science extend their resulting analysis across the whole domain of science. How then should a properly conceived deflationary or naturalistic philosophy of science address a given science?

Fine, Sismondo and the various proponents of normative naturalistic philosophy of science all offer, to one extent or another, answers to these questions. Fine seeks to characterise both the methods and goals of science according to the dictates of NOA with its realist-like demand for causal explanation and its non-realist view of theoretical entities. But Fine's view is inadequate because it is clear that NOA is not characteristic of most science and this defeats its deflationary credentials. Simondo's deflationary metaphysics on the other hand correctly recommends recognising the plurality that exists across the domain of science.

But what of NOA's anti-normative stance? Might it not be the case that although NOA's views on explanation and its anti-interpretative stance fail in many cases, limits can still be placed on the role of the philosophy of science in virtue of warnings against committing the naturalistic fallacy? Indeed, Fine's (1998) recent work has downplayed the specifics of NOA arguing instead for "procedural objectivity". He still sees the goal of science, however, as more or less fixed, i.e. the production of explanations. His procedural objectivity is only pluralist about the means by which specific sciences achieve "trust in [their] product" (Fine, 1998: 18). Normative approaches to interpretation of the realism question (even on a case by case basis) are still ruled out in this picture and Fine retains his hostility to interpretation of any science in terms of the realism question.

Laudan (1988; 1990) and Stump (1990; 1992) have both argued against such a ban on philosophy's normative role. Indeed, as Stump (1992: 458) has noted, a complete ban would imply the maintenance of a strict fact/value distinction. And this of course is impossible because it is impossible to isolate "facts" from theoretical assumptions about how they are to be interpreted, and these assumptions include values. This point can be fleshed out a little by way of an analogy with the failure of another traditional philosophical distinction, the logical empiricist's theoretical/observational distinction.

One of the main reasons for the failure of logical empiricist distinction between the observable and the unobservable parts of theoretical vocabularies is the fact that so-called strict observation terms receive their interpretation in part through theoretical (nonobservable) concepts. Scientific language is laden with theory, so it is impossible to isolate the strictly observational or factual. Facts then are never independent from theory. Just as this is true within science, so it is true of the study of science. So-called facts about a given science will be permeated with theory about what science is and ought to be. Given this, is it not reasonable to suppose that conclusions about how sci- 
ence can and does operate will influence conclusions about how science should operate and vice versa? In a somewhat related way Laudan (1990: 46) has argued for a normative naturalism on the basis that normative dictates of scientific epistemology can be seen as "hypothetical imperatives" that facilitate the realisation of certain goals. Thus, even though the goals and methods of science have changed over history, certain methods are appropriate for achieving certain goals. The validity of such imperatives can be scrutinised empirically by virtue of their relative success at achieving the desired goals. Facts about how science does operate, in Laudan's view, are predicated on values about how best to achieve the goals of science and value judgements about what those goals should be. For Laudan, facts uncovered by the philosopher of science about how science operates are permeated with value judgements about what the goals of science are and how these are best achieved.

These proposals from Stump and Laudan certainly make adequate response to the critics of the prospects for a normative role for a deflationary philosophy of science. Moreover, Laudan has offered some interesting proposals on the nature and function of normative principles that the philosophy of science can identify and deploy. However, Laudan's position while certainly superior to Fine's is still inferior to Stump's and Sismondo's in that it is insufficiently pluralistic. While Laudan does recognise that the methods and goals of science have changed over history, he makes no note of the vast plurality that exists within science at any given time (Stump, 1992: 457). As Sismondo has noted in his critique of NOA, failing to take into account the real plurality of various sciences' different stands on interpretation fails to do justice to science as philosophy finds it. Doing justice to science as philosophy finds it is supposed to be part of the point of Laudan's naturalistic position. After all, a non-pluralist naturalism is just as inadequate as a non-naturalist position. Nevertheless, Laudan's view does contain valuable lessons for the naturalist; in particular his suggestions about how normative principles are to be formulated, deployed and judged.

For Laudan, epistemological principles play a normative role in so far as they serve as hypothetical imperatives (valid in so far as they are successful) that serve as recommendations on how to proceed methodologically. Likewise, lessons can be drawn from his proposal that the methodological dictates of a given scientific programme can be judged on the basis of their relative success in achieving their specific goals. The interpretation of the realism question (as the Scott case shows) plays a methodological role as well as dictating the goals of science (e.g. explanation or prediction). The respective stance to the realism question on the part of a science, then, can be seen as part of the source of these same hypothetical imperatives. These are judged to be successful or not on the basis of their success in achieving their stated aims.

While these proposals are certainly not inconsistent with Stump's quite general proposals or Sismondo's treatment of Scott's programme, neither of these accounts fully develops the normative potential latent in deflationary or naturalised philosophy of science. Sismondo's 
view tends to leave the normative role as an implicit possibility. Stump, on the other hand, does directly argue that such a normative role is possible. Nevertheless, neither his nor Sismondo's account directly treats the question of how this role for the philosophy of science is to be deployed in cases where foundational debates on goals, interpretation and methods are taking place within a specific scientific programme. How exactly is this adjudication to take place? At a later point I will answer this question by developing an account of the normative role that I am arguing for by deploying it in a specific case study.

As I have just noted, in spite of the fact that Sismondo's account of a deflationary philosophy of science is appealing in many respects, it does not by itself supply an account of how competing stances to the realism question are to be adjudicated within a given scientific programme. In the example of Scott's programme discussed above, Sismondo deployed a constructivist philosophy of science to account for Scott's practice but left the question of the success of that approach to the judgement of the reader and of history. Sismondo's account as far as it goes, then, deploys the deflationary approach to characterise and account for the practice and nature of the scientific endeavour after the fact. But if a philosophy of science is to have anything but a descriptive role, it ought to be able to help adjudicate the more plausible stance in a given situation at any given time. I contend that philosophy can indeed play such a role.

In the next sections of this paper I address the question of the adjudication of the correct stance to the realism question directly. I argue that the stance on the realism question taken by a research programme will come hand in hand with methodological maxims that will play a role in directing the course and goals of that programme. The philosopher of science can identify these maxims along with their relation to philosophical goals and their practical import. Thus, the adjudication of the correct stance to the realism question in any given situation proceeds in so far as any given stance will imply methodological maxims that will bear practical fruit. These maxims (and the stance to the realism question that they imply) can be judged against their respective success at achieving their stated goals. To illustrate this point the last section will develop both the descriptive and normative role for deflationary philosophy of science. This will take place in the context of a discussion of quantum mechanics as a case study. Before this takes place, however, I will provide a brief overview of the general form of the deflationary approach that has emerged from the discussion of Sismondo, Stump and Laudan's proposals.

\section{Normative Deflationary Metaphysics}

For a supporter of NOA the role of philosophy is very limited. Indeed, philosophy for NOA must leave science as it is. Any additional content would "add something" to our understanding that is not to be found in science itself and is therefore to be avoided. For NOA a scientific research programme can be neither understood nor judged on the basis of philosophical considerations. For NOA, there is nothing for philosophy to do. As Sismondo has pointed out, however, NOA fails to take its own advice in 
all case by sometimes ignoring the very real interpretative aspects of science. One way to understand the reason for this failure is to consider it in the light of the reasons for the failure of the early formulations of logical empiricism. The cornerstone of the early logical empiricist programme was the search for demarcation between scientific (cognitively significant) statements and metaphysical statements. This project was eventually abandoned as untenable for reasons that are related to the realist rejection of the distinction between observable and theoretical content in scientific theories. How is NOA's stance to be sustained in the absence of a clear distinction between the scientific and the metaphysical? As both NOA and the realists argue, it is not to be found in any a priori philosophical approach, and as I have argued above, no clear distinction is to be found in science either. Where then is NOA to find support for its antimetaphysical (i.e. its anti-interpretative) stance?

It would seem that the correct lesson to be learned from the failure of the first phase of logical empiricism is that there simply is no clear distinction to be drawn. Speaking in another context, this point is well expressed by the philosopher of economics, Alexander Rosenberg,

What will [scientific] theories contain?
The material findings of science natu-
rally, but philosophical, logical, episte-
mological and metaphysical theses will
be included as well (Rosenberg, 1992:
11). ${ }^{1}$

The position I have adopted seems to take this lesson to heart in a way in which NOA does not. In this view, a deflationary account of science leaves considerable room for interpretation on the part of both scientists and philosophers of science. The main feature of a workable deflationary view is the demand that the individual sciences must be analysed in their own contexts, and this must include taking their identifiably metaphysical aspects (i.e. stance to the realism question) seriously as well as their empirical aspects. Given the overlap between science and philosophical interpretation, there is considerable latitude for the philosophy to play a role in several areas relating to scientific practice. These include a role in the construction of the conceptual foundations of research programmes. Philosophy can thus play a role in the adjudication of the correct stance of a programme with regards to its conceptual foundations.

A position like the one I have been developing in this paper can only really be illustrated trough the use of case studies. This is so because the actual process of constructing an interpretation of any given scientific programme will be unique to each individual case, even if there will be large groups that will share fairly similar analyses. This is so for the obvious reason that any explicit outline of a procedure that is to be applied to all cases would do violence to the deflationary dictum that specific practice must effect the philosophical approach. Too rigid procedures that are supposed to apply in all cases might gloss over the genuine differences that exist across the sciences. Nevertheless, it is worthwhile to pause before considering a case study to outline the general features that characterise the approach I favour.

Perhaps the most important feature of the deflationary position I am arguing for is borrowed from Sismondo. This is the observation that different pro- 
grammes have different interpretative approaches and that these approaches have methodological implications. When approaching a programme like quantum mechanics or Scott's study of lab mice, how should a philosopher of science proceed when trying to identify the correct stance? The most straightforward approach is to first proceed by identifying the general goal of the research and of course by looking at the means by which that goal is achieved. This is how Sismondo proceeded when dealing with Scott's work. Questions, however, remain. How is the philosopher to approach the question of adjudicating the relative success of the approach in question and how are competing interpretations that exist within a programme to be adjudicated?

To address these questions lessons can be learned from the naturalist philosophies of science. In order to avoid imposing a philosophical a priori stance on science, a science ought to be approached using the methods of science itself. However, the methods and the goals of the sciences differ. How is the philosopher to choose? To fail to adopt a pluralistic stance amounts to the same thing as adopting an a priori philosophical interpretation. Indeed, Stump has pointed out that Laudan's chief trouble is that he tends to take science as a monolithic entity with regards to its goals at any given point in time. In this sense, adopting Laudan's position at face value is not to be recommended. There are however some quite specific proposals in Laudan's normative naturalism that can be adopted to answer the questions that Sismondo and Stump do not directly address.

Laudan argues that philosophical principles found in science play a normative role in so far as they serve as recommendations on how to proceed methodologically by providing both goals and hypothetical imperatives on how those goals are to be achieved. For Laudan the success of a philosophical approach (and the validity of its goals) can be adjudicated on the basis of the relative success of the deployment of those hypothetical imperatives in practice. For example, a science that takes as its goal the causal explanation of its studied phenomena will deploy a realist metaphysics in order to provide guidelines in the form of hypothetical imperatives about how to achieve that goal. One such guideline will be this: if causal explanation is the goal, then a theory that simply provides an empirically adequate model should not be seen as complete. As I will demonstrate in the next section, this is exactly how the "hidden variables" interpretation of quantum mechanics should be read. Its relative success (or lack thereof) can be appreciated through the adjudication of the deployment of that guideline in practice in that case. Thus, the philosopher of science should identify the hypothetical imperatives provided by a science's stance to the realism question that dictate how the stated goals associated with that stance are to be achieved. Looking at the relation between philosophy and methodology in this way can facilitate the adjudication of a research programme. The work of Sismondo and Stump, however, reminds us that these goals and imperatives will vary, and approaching science with a preconceived idea of what its goals and methods are will not do justice to a science in the way that Laudan's naturalism promises. 
From a pluralist perspective, the position I am developing takes its normative role in a manner which otherwise follows Laudan's closely. The philosophical approach of a science will imply both the goals of a science and methodological maxims in the form of hypothetical imperatives on how to achieve those goals. The success of the deployment of those maxims lends weight to both the proposition that the goal in question is worthwhile and to the proposition that the methods to attain it are to be recommended. However, the version of the deflationary approach that we ought to adopt should also be able to adjudicate between competing interpretative positions that co-exist within a single science.

In this situation too, the proposals borrowed from Laudan can help answer this question. Again, however, Laudan's own formulation is not fully adequate because it is insufficiently pluralist. Laudan would have to proceed in such a case by simply recommending what he takes to be the approach supported by current science. For this reason it would fail to do justice to the particularities of the case in question by denying the interpretative and methodological pluralism that exists across and within the sciences.

My proposal in such a situation is to proceed instead by way of a comparative evaluation. Both approaches should be analysed on their own terms by identifying both the goals and methods of achieving those goals recommended by the interpretation in question. The relative success of each approach can then be compared. The approach that is more successful on its own terms is the one to be recommended by general philosophy of science. The example of the "hidden variables debate" in quantum mechanics will illustrate this proposal fully.

\section{Deflationary Metaphysics and Quantum Mechanics}

There is, of course, a vast literature on the conceptual difficulties associated with quantum mechanics (henceforth QT) and it is beyond the scope of this paper to deal with them all. I restrict this discussion to showing how the realism issue has had a large and direct impact on the practice in the field. This demonstration shows that the if the dictum that individual sciences are to be approached within their own context is sound then this implies dealing with explicitly philosophical considerations. In order to do this with brevity, I focus on the philosophical positions on the realism question associated with two interpretations prevalent in the field, the (stochastic) hidden-variables interpretation and the Copenhagen interpretation. This discussion focuses only on philosophical issues directly related to realism and avoids technical considerations as much as possible. I proceed in this manner in order to prevent too lengthy a digression from the main theme of this paper and because a more detailed treatment of the technical issues is simply not possible within the broad format of this discussion. For the same reasons I also restrict this discussion primarily to non-relativistic quantum mechanics. That is, I do not treat in any great detail the philosophical problems associated with quantum field theory. Where reference quantum field theory is necessary I restrict the discussion only to those features that bear upon the debate over the 
interpretation of standard quantum mechanics (QT).

\section{Copenhagen vs. "Hidden Variables"}

Essentially quantum theory provides results that specify the probability of the dynamical quantities in a quantum system (like "spin") having particular values when measured (according to the "Born rules"). However, the interpretation of what this means exactly has been a matter of considerable controversy. For example, do the Born rules specify the probability of a quantity having a specific value or do they specify that the quantity acquires this quantity on measurement? Do they simply relate to the probability that the measurement apparatus will record a particular value? Further questions arise from the nature of quantum mechanical description of the phenomena that it deals with. Does quantum mechanics describe the micro-systems that it deals with? That is, does it describe (however abstractly) and explain the causal interactions taking place in the micro world or does it simply restrict itself to the description (and prediction) of what is observed in experimental situations ( $c f$., Healey, 1999: 9$11)$ ?

These considerations have vexed not only philosophers but also scientists, both in terms of foundational interpretation but also in terms of practice. While many difficulties are associated with quantum mechanics, one of the most important arises from the commonly held realist conviction that science not only derives predictions about observed results but must also provide causal explanations of what is observed. If this demand for explanation is valid then a satisfactory causal interpretation of the system that underlies the experimental observations must be given. This is so because a causal explanation of the reason why measurements have the results that they do entails a complete description of the physical system underlying the measured result. For the realist an unresolved interpretation of the system underlying the measurements leaves quantum mechanics incomplete, given that this unresolved question leaves us without a causal explanation for the observed outcomes. On the other hand, for an anti-realist like van Fraassen who denies that the demand for explanation (causal or not) is always present, quantum mechanics can be easily seen to be complete if it is just a predictive tool and not an explanatory device. ${ }^{2}$

It should be noted, however, that some realists have tried to soften their positions somewhat to accommodate quantum mechanics. Some, like McMullen (1991), claim that the demand for a causal reading of the term "explanation" can be dropped in selective cases without jeopardising a general realist stance. This puts McMullen, however, in an awkward position. If the demand is dropped in this case, why not the general demand for a realist causal view of explanation, or even the basic requirement that science always seek explanations? One major problem is the demand that science must be explanatory (either taken in realist or NOA's terms), in this sense McMullen's view, like NOA, suffers from the problems that plague any $a$ priori stance.

The question of the completeness of quantum mechanics has obvious methodological ramifications because the answer that a particular scientist favours 
will dictate whether or not she views the science as satisfactory as it is or as requiring fundamental reform that necessitates a great deal of foundational research. Quantum physics split early on into several camps on exactly this issue with the realist demand for causal explanation being famously championed by Einstein and the anti-realist rejection of this demand deriving from the now dominant "Copenhagen Interpretation" associated with Heisenberg and Bohr.

\section{Empirical Dividends of the Debate}

This methodological debate over the realist demand for explanation has also yielded empirical dividends. This can be demonstrated through a very brief examination of the much discussed "Einstein, Podolsky, Rosen Paradox" (EPR). The following brief account, borrowed from van Fraassen, highlights the salient points of EPR that are germane to this discussion. One way to describe the EPR thought experiments is to consider an initial state $S$ that undergoes a change into a new state characterised by the attributes $F$ and $G$. $F$ and $G$ are not individual attributes but sets of attributes such that if the $F$ attribute is $F_{1}$ then the $G$ attribute will be $G_{1} . F_{2}$ will correspond with $G_{2}$ and so on. The physical situation that this is usually related to is the production of a particle pair such that the total spin of the system is 0 i.e. if the spin of particle $F$ is $1 / 2$ then the spin of $G$ will be $-1 / 2$ and so on. However, the particular $F n / G n$ correlation that results occurs indetermanistically. Thus, $n$ will not be caused, and the correlation $F n / G n$ will not have a common cause if $S$ is a complete description of the initial state (van Fraassen, 1980: 29). For the realist, of course, correlations like those in EPR stand in need of explanation, the uncaused $n$ in EPR is impossible and they argue that the quantum mechanical description of the initial state $S$ must be incomplete. This realist conviction has given rise to a wide variety of so called "hidden variable" theories that purport to explain the $F n / G n$ correlation. These theories have been tested empirically and it is now widely recognised that they are empirically inferior to their anti-realist rivals, at least in the case of hidden variable theories that preserve relativity theory's ban on "non-local" effects (van Fraassen, 1974; 1980; Fine, 1986: 151-71; Healey, 1989: 140).

\section{"Non-local" Hidden Variables and their Future}

A supporter of hidden variable theory could of course point out that there are "non-local" approaches to a hidden variable programme that can account for the EPR experiments. For example, Cushing (1994) and others have argued that the "non local" approaches associated with David Bohm and others can be shown to be empirically equivalent to the antirealist inspired interpretations. To address this debate in any great detail would be somewhat digressive since the purpose of this chapter is not to provide a full account of every position possible in the philosophy of QT, but to use QT to show how competing positions are to be adjudicated in context. In any event, this realist move admits that Copenhagen style anti-realism is at least a superior option to local hidden variable theories, and this is all that my argument requires. Nevertheless, this possible turn in the realism debate does warrant some com- 
ment.

Even if an interpretation like Cushing's were to eventually show itself empirically superior this would neither tell against my arguments nor imply a general adoption of realism as the stance for all science. It would only imply the success of a realist inspired methodology in the case of QT, and this of course is fine from the deflationary point of view that I defend in this paper. It would however rob the anti-realists of one key example of a science that functions perfectly well without the realist demand for explanation.

Nevertheless, there are some good reasons to entertain grave doubts about the "non-local" programmes. For one thing they are very difficult to reconcile with relativity theory, given their nonlocal effects. While this need not rule them out $a$ priori, these approaches do imply that the demand for causal explanation in quantum mechanics does do violence to at least the spirit of relativity theory. This is because attempts to make them consistent with special relativity imply the introduction of a preferred frame of reference and it is generally considered to be a triumph of special relativity that no such preferred frame of reference is required. This is difficult for the realist because realism demands more than causal explanation, it also demands that different theories be consistent (a realist can hardly defend the truth of two incompatible theories). Moreover, non-local approaches involve the belief in a number of theoretical entities for which there is at present no evidence (quantum potentials, pilot waves, "the subquantum ether" etc., depending on the non-local approach in question). It is simply not clear that giving up the realist demand for explanation in this one case is a higher price to pay. This becomes more telling when one considers the fact that unlike the local hidden variable theories, no feasible experiments currently exist that could potentially show the non-local approaches to be empirically superior (Healey, 1989: 24-6). Bohm's interpretation is certainly not the simpler of the two and it performs no better.

Cushing's case for Bohm's approach becomes worse when one takes the issue of fruitfulness into account. The standard interpretation of QT arose alongside newer and strikingly successful theories like quantum electrodynamics and its successor quantum field theories, which like QT eschew a causally descriptive general methodology. Realist interpretations of QT (in general) are much more difficult to reconcile with quantum field theory than they are with ordinary QT. As Teller (1990) has pointed out, mathematical techniques central to quantum field theory like the process of renormalisation of infinite divergent integrals cannot be equated with a causal description of the actual system without introducing considerable conceptual difficulties. For example, in some cases the bare mass of a "particle" before interaction with its own field would have to be taken as infinite and so also must be the value of its interaction with its field (Teller, 1990: 76). One is hardly required to believe in a particle having infinite mass before interaction if one takes the formalism of renormalisation to be simply a calculating device used to generate empirically adequate predictions. It is very difficult to see how a demand for realist causal explanation can accept the use of a technique like re- 
normalisation to be legitimate while at the same time retaining Bohmian QT's most attractive feature, its purported explanatory fruitfulness. I am not, of course, claiming that Bohmian QT cannot mathematically admit renormalisation, only that it poses difficulties for the appealing explanatory features of Bohmian mechanics. Given all of this, it becomes obvious that the Copenhagen interpretation poses no new conceptual problems for the relativistic extension of QT that takes place in quantum field theory, but the Bohm interpretation is not quite so fruitful. A very strong argument indeed exists to take non-local realist approaches to QT to be inferior in practice to the standard interpretation, at least at present.

A quantum realist could still hold out hope that someday Bohm's approach could overcome the conceptual difficulties posed by quantum field theory and that some experiment showing its empirical superiority might someday be devised. However, even if such experiments could be conducted (and this is not at all likely) then it would serve as an excellent example of how a philosophical standpoint imposes methodological demands that can bear empirical fruit, whichever way the experiments come out. For the time being, then, it is not unreasonable to take the Copenhagen Interpretation to be the most successful and fruitful to date. Certainly this opinion is the majority stance among working physicists who concern themselves with foundational debates. Thus, while it must be admitted that a realist approach to quantum mechanics has not been completely ruled out, for the time being it would not be unreasonable to conclude that scientific practice has, in this case, so far tended to support and indeed profit from an anti-realist stance.

\section{Quantum Theory and Deflationary Philosophy of Science}

In any event, even if subsequent work in the field forces a reversal of the conclusions stated at the end of the last paragraph, the failure of the local-hidden variable approaches still serves as an instructive example of the deflationary approach in action. In these instances the methodological goals of the rival positions derive in large part from the respective stances they take to the realism question. The superior stance in this case emerged from the empirical fruit that resulted from their relative success. That is to say, when everything is taken into account, the Copenhagen interpretation has been superior at accomplishing its methodological goals relative to its rivals. It has proved better at providing what it promises (empirically adequate predictions) than the local hidden variables approach has been at providing what it promises (causal explanations).

The Copenhagen vs. hidden variables debate shows how the stance on the realism question is settled in context. In the sort of deflationary philosophy of science that I am defending in this paper, nothing implies that the correct stance need remain static permanently. Just as science is not a monolithic entity with one correct methodological approach and stance on the realism question, variability exists within specific research programmes over time. Nevertheless, the best stance can be empirically adjudicated. Indeed, the adoption of anti-realism in fundamental physical 
theory that the advent of quantum mechanics brought about can be seen as one such instance. In previous centuries much of physics had been characterised by a robustly realist stance. But this situation changed in the early twentieth century with the availability of credible anti-realist philosophies and the strange experimental results of early quantum research. These features combined to make such a shift plausible and in fact prudent. It would be foolhardy to assume that the situation could never reverse itself.

The point that I am making in this discussion is not that a realist approach is always a failure or that the realist methodological dicta are always to be rejected. As I already stressed, I am not arguing for any particular $a$ priori methodological or philosophical conception of science in general. What I am trying to make clear is this: the correct lesson to be drawn from the failure of realist inspired approaches to QT is that the most general methodological dictates of scientific theories derive in part from their philosophical stance. There have of course been a wide variety of scientific theories that have seen empirical triumphs arise from the realist methodological commitment to the demand for causal explanation.

The case of the empirical success of the Copenhagen interpretation shows that the general methodological stance can lead to the formation of different empirical interpretations of the same laboratory situation. In the case of EPR, scientists of a realist bent formulated and gave their support to the local hidden variable theories, which made different predictions than their anti-realist rivals, and the debate was settled empiri- cally. So not only does the stance associated with a theory make methodological demands on its adherents, the success or lack of success of the practical deployment of those dictates allows judgement to be passed on the validity of those demands. These judgements cannot be generalised to the whole corpus of science but come on a case by case basis. This should be no surprise if philosophical theses are part and parcel of scientific theories. The domain of a theory's stance to the realism question is the domain of that theory and no more. The validity of its methodological dictates can be judged on the basis of the success of their deployment in practice relative to their rivals.

The example of quantum mechanics illustrates the role that philosophical approaches to the realism debate have for general scientific methodology. The individual positions within a field each have philosophical content and their own stance with regards to realism. This content makes demands that take the form of methodological maxims. In the case of the "hidden variables" theories we can see the realist maxim "seek causal explanations for observed regularities" at work. In the Copenhagen Interpretation it is easy to see the methodological maxim, “don't worry about hidden causes, focus on what can be observed because there is nothing else to discuss" at work. This of course is in line with the idea that the quest for empirical adequacy is primary and not the quest for causal explanation. The adjudication of the correct stance came in the form of the success of the Copenhagen Interpretation relative to its local-hidden variable rivals. While this example does not settle either the realism question or the 
proper methodological stance for every science it shows how the debate has methodological import in terms of formulating philosophically derived maxims that guide research. The realism debate is to be settled on a case by case basis in terms of the relative success or failure of the deployment of the methodological maxims that are recommended by the various sides. And it can fall to the philosopher to interpret the stance to the realism question implicit in research programmes and to derive the implicit methodological maxims.

When science is taken within its own context it becomes clear that the line between science and philosophy is a blurry one and that science at its most general becomes clearly philosophical. Philosophy, then, has an important role in the formation of the methodological dictates associated with scientific theories. More significantly, this role, as the EPR case demonstrates, makes it possible for the adjudication of the best philosophical and methodological stance to take place on a case by case basis. Thus, a pluralist deflationary philosophy of science permits an understanding of the methodological role that philosophy plays in scientific practice, an appreciation that NOA fails to provide.

\section{Acknowledgements}

I would like to thank Idil Boran, Jean Leroux and Sergio Sismondo for their helpful suggestions with earlier versions of this paper.

\section{Notes}

1 I am not arguing here that Rosenberg would support deflationary philosophy of science like the one I propose. On the contrary he argues that theories are to be judged on the basis of their predictive track record. He also rejects pluralism with regards to the goals of science. For him the general goal of all science is "knowledge". Laudan (1990: 49) has pointed out that in addition to suffering from the faults of any universalist characterisation, this proposal is unhelpfully vague. McArthur (1997; 1999) also provides a critique of Rosenberg's work.

2 Van Fraassen's view of quantum mechanics is somewhat subtler than the way it is expressed in the text. For van Fraasen there is no actual ban on trying to model and explain unobserved events. However, such unobserved causal features go in the unobservable parts of theoretical models and are not to be taken realistically. An empirically superior hidden-variables approach might well be acceptable to van Fraassen, but there are currently none that are superior to the interpretation van Fraassen favours.

\section{References}

Crasnow, S.

2000 "How Natural Can Ontology Be?" Philosophy of Science, 67, 114-122.

Cushing, J.T.

1994 Quantum Mechanics. Chicago: University of Chicago Press

Fine, A.

1986 The Shaky Game; Einstein Realism and the Quantum Theory. Chicago: The University of Chicago Press.

1991 "Piecemeal Realism.” Philosophical Studies 61, 76-96.

1998 "The Viewpoint of No-One In Particular." Proceedings and Addresses of the American Philosophical Association, 72, 9-20. 
Healey, R.

1989 The Philosophy of Quantum Mechanics. Cambridge: Cambridge University Press, 1989.

Laudan, L.

1988 "Progress or Rationality? The Prospects for a Normative Naturalism." American Philosophical Quarterly, 24, 19-31.

1990 "Normative Naturalism." Philosophy of Science 57, 44-59.

McArthur, D.

1997 "What Good is the Philosophy of Science." Contemporary Philosophy 19, 11-16.

1999 "Evaluating Economics: an Examination of Alexander Rosenberg's Philosophy of Economics." Contemporary Philosophy 21, 43-52

McMullen, E.

1991 "Selective Anti-Realism." Philosophical Studies 61, 97-108.

Rosenberg, A.

1992 Economics- Mathematical Politics or Science of Diminishing Returns. Chicago: Chicago University Press.

Sismondo, S.

1997 "Deflationary Metaphysics and the Construction of Laboratory Mice." Metaphilosophy 28, 221-232.

Stump, D.

1990 "Fallibilism, Naturalism and the Traditional Requirements for Knowledge." Studies in the History and Philosophy of Science, 22, 451-469.

1992 "Naturalised Philosophy of Science with a Plurality of Methods." Philoso-

Teller, P. phy of Science 59, 456-460.

1990 "Three Problems of Renormalisation." Pp. 79-93 in Brown and Harré (eds.), Philosophical Foundations of Quantum Field theory. Oxford: Clarendon Press.

van Fraassen, B.

1974 "The Einstein-Podolski-Rosen Paradox." Synthese 29, 293-309.

1980 The Scientific Image. New York, New York: Oxford University Press.
Dan McArthur

Department of Philosophy, University of Regina,

Canada

daniel.mcarthur@sympatico.ca 